\title{
Reconstructing the properties of dark energy from recent observations
}

\author{
Puxun $\mathrm{Wu}^{a}$ and Hongwei $\mathrm{Yu}^{b, *}$ \\ ${ }^{a}$ Institute of Math-Physics and School of Sciences, \\ Central South University of Forestry and Technology, Changsha, Hunan 410004, China \\ ${ }^{b}$ Department of Physics and Institute of Physics, \\ Hunan Normal University, Changsha, Hunan 410081, China
}

\begin{abstract}
We explore the properties of dark energy from recent observational data, including the Gold Sne Ia, the baryonic acoustic oscillation peak from SDSS, the CMB shift parameter from WMAP3, the X-ray gas mass fraction in cluster and the Hubble parameter versus redshift. The $\Lambda C D M$ model with curvature and two parameterized dark energy models are studied. For the $\Lambda C D M$ model, we find that the flat universe is consistent with observations at the $1 \sigma$ confidence level and a closed universe is slightly favored by these data. For two parameterized dark energy models, with the prior given on the present matter density, $\Omega_{m 0}$, with $\Omega_{m 0}=0.24, \Omega_{m 0}=0.28$ and $\Omega_{m 0}=0.32$, our result seems to suggest that the trend of $\Omega_{m 0}$ dependence for an evolving dark energy from a combination of the observational data sets is model-dependent.
\end{abstract}

PACS numbers: 98.80.-k, 98.80.Es

* corresponding author 


\section{INTRODUCTION}

The present cosmic accelerating expansion has been confirmed by various observations, including the Type Ia Supernovae (Sne Ia) [1, 2, 3, 4, 5, 6], CMB [7, 8, 9, 10, 11] and large scale structure (LSS) [12, 13], etc. In order to explain this observed phenomenon, it is usually assumed that there exists, in the universe, an exotic energy component with negative pressure, named dark energy (see [14, 15, 16, 17] for recent reviews), which presumably began to dominate the evolution of the universe only recently. The simplest candidate of

dark energy is the cosmological constant $\Lambda[18,19,20,21]$. It fits the observational data very well, but at the same time, it also encounters two problems, i.e., the cosmological constant problem (why is the inferred value of cosmological constant so tiny (120 orders of magnitude lower) compared to the typical vacuum energy values predicted by particle physics?) and the coincidence problem (why is its energy density comparable to the matter density right now?). Therefore, some dynamical scalar fields, such as quintessence [22, 23, 24], phantom [25] and quintom [26], etc, are suggested as alternative candidates of dark energy. One of the features of these scalar field models is that their equations of state parameter, $w$, which embodies both gravitational and evolutionary properties of dark energy, is evolving with the cosmic expansion.

On the other hand, the growing number of dark energy models has prompted people to adopt a complementary approach, which assumes an arbitrary parametrization for the equation of state $w(z)$ in a model-independent way and aims to reconstruct the properties of dark energy directly from observations. Currently, there are many model independent parameterizations (see for example, [27, 28, 29, 30, 31, 32]). In general, using these parameterizations and the observational data, one can determine the present value of $w$ and whether it evolves as the universe expands, in particular, whether the phantom divide line (PDL) is crossed. In this regard, Nesseris and Perivolaropoulos [33] has used the Chevallier-Polarski-Linder parametrization $w(z)=w_{0}+w_{1} z /(1+z)$ [28] to explore the properties of dark energy with some observational data (including new Gold Sne Ia, SNLS Sne Ia, CMB, BAO, the cluster baryon gas mass fraction $(\mathrm{CBF})$ and $2 \mathrm{dF}$ galaxy redshift survey(2dFGRS) ) and found that the Gold data set mildly favors dynamically evolving dark energy with the crossing of the PDL while the SNLS does not, and the combination of $\mathrm{CMB}+\mathrm{BAO}+\mathrm{CBF}+2 \mathrm{dFGRS}$ mildly favors the crossing of PDL only for low values of $\Omega_{m 0}\left(\Omega_{m 0} \leq 0.25\right)$ prior consid- 
ered and with a higher prior matter density the evolving features of dark energy becomes weaker and weaker. Similar trend of $\Omega_{m 0}$ dependence was found using the model [34], $w(z)=\frac{1+z}{3} \frac{A_{1}+2 A_{2}(1+z)}{\Omega_{D E}}-1$, with the CMB and BAO. However, constraints from a combination of the supernovae and other observational data has not been analyzed in Ref. [33], and although that of the Sne and $\mathrm{CMB}+\mathrm{BAO}$ was examined in Ref. [34], but the marginalization was considered only for $\Omega_{m 0}=0.28 \pm 0.03$ prior. Therefore, it remains interesting to see what happens to the conclusions reached in Refs. [33, 34], when the combination of all observational data is analyzed for different $\Omega_{m 0}$ prior considered. The present paper aims to fill the gap. We discuss the constraints from the combination of different observational datasets. Besides the data sets of Sne Ia, BAO and CMB, in our analysis we add the datasets of the X-ray gas mass fraction in cluster and the Hubble parameter versus redshift. Firstly the $\Lambda C D M$ model with curvature is discussed. Then, two parameterized dark energy models: $w(z)=w_{0}+w_{1} z /(1+z)$ and $w(z)=\frac{1+z}{3} \frac{A_{1}+2 A_{2}(1+z)}{\Omega_{D E}}-1$, are studied to see if the properties of dark energy thus reconstructed are model-independent.

\section{THE OBSERVATIONAL DATA}

\section{A. The Gold Sne Ia data}

The Sne Ia data considered in this paper is the 182 Gold set. This set was released by Riess et al. [4] with a consistent and robust manner. It consists of 119 previously published data points [3], 16 points discovered recently by the Hubble Space Telescope (HST) and 47 points from the first year release of the SNLS dataset [5]. For these Sne Ia, the data is released with the form of distance modulus $\mu$, which is relative with the luminosity distance $d^{L}$ through

$$
\mu\left(z, H_{0}, p_{j}\right)=5 \log _{10}\left[d^{L}\left(z, p_{j}\right)\right]+\mathcal{M}
$$

Here for a flat universe $d^{L}=(1+z) \int_{0}^{z} d z^{\prime} / E\left(z^{\prime}, p_{j}\right)$ with $E^{2}(z)=H^{2}(z) / H_{0}^{2}=\Omega_{m 0}+$ $\left(1-\Omega_{m 0}\right) \exp \left[3 \int_{0}^{z} \frac{d z^{\prime}}{1+z^{\prime}}\left(1+w\left(z^{\prime}\right)\right)\right], \mathcal{M}=M-5 \log _{10}\left(H_{0}\right), H_{0}=100 h, M$ is the absolute magnitude of the object and $p_{j}$ denote the model parameters of dark energy. The constraints on the dark energy models from Sne Ia data can be obtained by the maximum likelihood 
method, so the best fit values for model parameters is determined by minimizing

$$
\chi_{\text {Sne }}^{2}\left(H_{0}, p_{j}\right)=\Sigma_{i} \frac{\left[\mu\left(z_{i}, H_{0}, p_{j}\right)-\mu_{o b s, i}\right]^{2}}{\sigma_{\mu_{o b s ~}, i}^{2}}
$$

\section{B. The baryonic acoustic oscillation peak}

From the large scale correlation function of luminous red galaxy in the Sloan Digital Sky Survey (SDSS), Eisenstein et al. [13] found a baryonic acoustic oscillation peak, which is consistent with the prediction from the acoustic oscillation in the primordial baryon-photon plasma at the recombination. Thus this peak remarkably confirms the Big Bang cosmology. Meanwhile it also provides a ruler to constrain the dark energy models, which can be used usually by a dimensionless parameter $A$,

$$
A\left(p_{j}\right)=\frac{\sqrt{\Omega_{m 0}}}{E\left(z_{1}, p_{j}\right)^{1 / 3}}\left[\frac{1}{z_{1}} \int_{0}^{z_{1}} \frac{d z}{E\left(z, p_{j}\right)}\right]^{2 / 3},
$$

for a flat universe, where $z_{1}=0.35$ and $A$ is measured to be $A=0.469 \pm 0.017$. The parameter $A$ is model-independent and clearly independent of the value of $h$ too. By minimizing

$$
\chi_{B A O}^{2}\left(p_{j}\right)=\frac{\left[A\left(p_{j}\right)-0.469\right]^{2}}{0.017^{2}}
$$

we can obtain the constraints from BAO.

\section{The CMB shift parameter}

For the CMB data, we use the shift parameter $R$ to research the properties of dark energy, which can be expressed as [35]

$$
R\left(p_{j}\right)=\sqrt{\Omega_{m 0}} \int_{0}^{z_{r}} \frac{d z}{E\left(z, p_{j}\right)},
$$

for a flat universe, where $z_{r}=1048\left[1+0.00124\left(\Omega_{b} h^{2}\right)^{-0.738}\right]\left[1+g_{1}\left(\Omega_{m 0} h^{2}\right)^{g_{2}}\right], \quad g_{1}=$ $0.078\left(\Omega_{b} h^{2}\right)^{-0.238}\left[1+39.5\left(\Omega_{b} h^{2}\right)^{0.763}\right]^{-1}$ and $g_{2}=0.56\left[1+21.1\left(\Omega_{b} h^{2}\right)^{1.81}\right]^{-1}[36]$. To calculate $z_{r}$, we let $\Omega_{b} h^{2}=0.024$. The results of three-year WMAP data [11] give $R=1.70 \pm 0.03$ [37]. Let us note that the quantity $R$ from CMB measurement is dependent on the value of $\Omega_{m 0} h^{2}$, thus when using this parameter it is required to marginalize over $\Omega_{m 0} h^{2}$ or assign some specific value to $h$. In our discussion we give a prior value $h=0.72$. We then place constraints on cosmological models using this shift parameter by minimizing

$$
\chi_{C M B}^{2}\left(p_{j}\right)=\frac{\left[R\left(p_{j}\right)-1.70\right]^{2}}{0.03^{2}} .
$$




\section{The baryon gas mass fraction of galaxy cluster}

Under the basic assumption: the baryon gas mass fraction in cluster is constant, a comparison of the gas mass fraction of galaxy clusters, $f_{\text {gas }}=M_{\text {gas }} / M_{\text {tot }}$, can be used to constrain the cosmological models. Following Allen et al. [38, 39] we fit the $f_{\text {gas }}$ data to a model described by

$$
f_{\text {gas }}^{\text {mod }}\left(z, p_{j}\right)=\frac{b \Omega_{b}(2 h)^{3 / 2}}{\left(1+0.19 h^{1 / 2}\right) \Omega_{m 0}}\left[\frac{d_{A}^{S C D M}}{d_{A}^{\text {mod }}\left(z, p_{j}\right)}\right]^{3 / 2},
$$

where $b$ is a parameter motivated by gas dynamical simulations, $d_{A}=d^{L} /(1+z)^{2}$ is the angular diameter distance, $d_{A}^{S C D M}$ is the angular diameter distance corresponding to the standard cold dark matter (SCDM) universe $\left(\Omega_{m 0}=1\right.$ for a flat universe). Following Nesseris and Perivolaropoulos [33], we define $\lambda=\frac{b \Omega_{b}(2 h)^{3 / 2}}{\left(1+0.19 h^{1 / 2}\right) \Omega_{m 0}}$ and treat it as a nuisance parameter. Using the theoretical method given in [33] to marginalize over $\lambda$, we can obtain the constraints by minimizing

$$
\chi_{f_{g a s}}^{2}\left(p_{j}\right)=C-\frac{B^{2}}{A},
$$

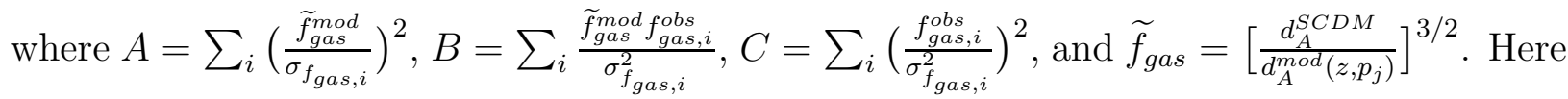
26 cluster data points given in Ref. [39] are used.

\section{E. The Hubble parameter data}

Based on differential ages of passively evolving galaxies determined from the Gemini Deep Deep Survey [40] and archival data [41] at redshift $0 \lesssim z \lesssim 1.8$, Simon et al. [42] obtained 9 data points of $H(z)$ at redshift $z_{i}$, which can be used to test the cosmological models by minimizing

$$
\chi_{H}^{2}\left(H_{0}, p_{j}\right)=\Sigma_{i} \frac{\left[H_{o b s}\left(z_{i}\right)-H_{t h}\left(z_{i}, H_{0}, p_{j}\right)\right]^{2}}{\sigma_{H i}^{2}},
$$

Recently these 9 Hubble parameter data points have been studied extensively by many authors [42, 43], however it doest not provide a tight constraint on dark energy models.

Thus the constraints on cosmological models from a combination of above discussed observational datasets can be obtained by minimizing

$$
\chi^{2}\left(H_{0}, p_{j}\right)=\chi_{\text {Sne }}^{2}\left(H_{0}, p_{j}\right)+\chi_{B A O}^{2}\left(p_{j}\right)+\chi_{C M B}^{2}\left(p_{j}\right)+\chi_{f g a s}^{2}\left(p_{j}\right)+\chi_{H}^{2}\left(H_{0}, p_{j}\right) .
$$


Since we are interested in the model parameters, $H_{0}$ becomes a nuisance parameter and is marginalized by a theoretical method given in Ref. [44] in calculating $\chi_{\text {Sne }}^{2}$ and $\chi_{H}^{2}$.

\section{RESULTS}

The $\Lambda C D M$ model with curvature is firstly discussed with the observational data. The results are shown in Fig. 1 for a combination of the above discussed observational data sets. At a $95.4 \%$ confidence level we obtain $\Omega_{m 0}=0.29_{-0.04}^{+0.04}$ and $\Omega_{k 0}=-0.016_{-0.029}^{+0.030}$ with $\chi^{2}=196.8$. It is easy to see that a spatially flat universe is consistent with the observations at a $68 \%$ confidence level and a closed universe is somewhat favored by these data sets.

TABLE I: The best-fit data of Mod1 with prior value $\Omega_{m 0}$. In the Table $\mathrm{G}+\mathrm{C}+\mathrm{B}$, $\mathrm{G}+\mathrm{C}+\mathrm{B}+\mathrm{f}$ and $\mathrm{G}+\mathrm{C}+\mathrm{B}+\mathrm{f}+\mathrm{H}$ represent the $\mathrm{Gold}+\mathrm{BAO}+\mathrm{CMB}$, Gold $+\mathrm{BAO}+\mathrm{CMB}+$ fgas and Gold $+\mathrm{BAO}+\mathrm{CMB}+$ fgas $+\mathrm{H}(\mathrm{z})$ respectively.

\begin{tabular}{|c|ccc|ccc|ccc|}
\hline & \multicolumn{3}{|c|}{$\Omega_{m 0}=0.24$} & \multicolumn{3}{c|}{$\Omega_{m 0}=0.28$} & \multicolumn{3}{c|}{$\Omega_{m 0}=0.32$} \\
\hline & $w_{0}$ & $w_{1}$ & $\chi_{\text {Min }}^{2}$ & $w_{0}$ & $w_{1}$ & $\chi_{\text {Min }}^{2}$ & $w_{0}$ & $w_{1}$ & $\chi_{\text {Min }}^{2}$ \\
\hline Gold & $-1.28_{-0.63}^{+0.59}$ & $2.64_{-3.14}^{+3.08}$ & 156.5 & $-1.38_{-0.72}^{+0.67}$ & $2.75_{-3.73}^{+3.52}$ & 156.5 & $-1.49_{-0.85}^{+0.79}$ & $2.81_{-4.62}^{+4.13}$ & 156.6 \\
Gold+BAO & $-1.45_{-0.55}^{+0.52}$ & $3.32_{-2.63}^{+2.79}$ & 158.1 & $-1.30_{-0.59}^{+0.53}$ & $2.43_{-3.40}^{+3.07}$ & 156.8 & $-1.11_{-0.64}^{+0.65}$ & $1.25_{-4.28}^{+3.50}$ & 160.7 \\
$\mathrm{CMB}+\mathrm{BAO}$ & $-1.47_{-0.69}^{+0.85}$ & $1.48_{-5.18}^{+1.51}$ & 0.001 & $-1.01_{-0.71}^{+0.94}$ & $0.61_{-6.08}^{+1.40}$ & 0.001 & $-0.60_{-0.71}^{+1.05}$ & $-0.37_{-6.75}^{+1.82}$ & 0.001 \\
$\mathrm{Gold}+\mathrm{CMB}$ & $-0.90_{-0.37}^{+0.42}$ & $0.27_{-1.98}^{+1.00}$ & 161.8 & $-1.05_{-0.40}^{+0.44}$ & $0.81_{-2.08}^{+0.81}$ & 158.6 & $-1.22_{-0.56}^{+0.43}$ & $1.28_{-1.78}^{+0.73}$ & 157.4 \\
$\mathrm{G}+\mathrm{C}+\mathrm{B}$ & $-1.04_{-0.31}^{+0.35}$ & $0.63_{-1.62}^{+0.79}$ & 165.1 & $-1.05_{-0.34}^{+0.39}$ & $0.79_{-1.91}^{+0.74}$ & 158.6 & $-1.06_{-0.36}^{+0.44}$ & $0.96_{-2.24}^{+0.68}$ & 160.8 \\
$\mathrm{G}+\mathrm{C}+\mathrm{B}+\mathrm{f}$ & $-1.07_{-0.30}^{+0.35}$ & $0.68_{-1.71}^{+0.76}$ & 191.1 & $-1.10_{-0.32}^{+0.40}$ & $0.88_{-2.08}^{+0.71}$ & 185.9 & $-1.13_{-0.35}^{+0.47}$ & $1.10_{-2.54}^{+0.62}$ & 189.6 \\
$\mathrm{G}+\mathrm{C}+\mathrm{B}+\mathrm{f}+\mathrm{H}$ & $-1.07_{-0.30}^{+0.30}$ & $0.71_{-1.42}^{+0.73}$ & 200.2 & $-1.09_{-0.32}^{+0.36}$ & $0.85_{-1.84}^{+0.70}$ & 194.9 & $-1.12_{-0.35}^{+0.47}$ & $1.06_{-2.54}^{+0.65}$ & 199.1 \\
\hline
\end{tabular}

Then we study, in the spatially flat case, the observational constraints on the following two-parameter models considered in Ref. [33] and [34] respectively.

$$
\begin{gathered}
\operatorname{Mod} 1: \quad w(z)=w_{0}+w_{1} \frac{z}{1+z}, \\
\operatorname{Mod} 2: \quad w(z)=\frac{1+z}{3} \frac{A_{1}+2 A_{2}(1+z)}{\Omega_{D E}}-1,
\end{gathered}
$$


TABLE II: The best-fit data of Mod2 with prior value $\Omega_{m 0}$. In the Table $\mathrm{G}+\mathrm{C}+\mathrm{B}$, $\mathrm{G}+\mathrm{C}+\mathrm{B}+\mathrm{f}$ and $\mathrm{G}+\mathrm{C}+\mathrm{B}+\mathrm{f}+\mathrm{H}$ represent the $\mathrm{Gold}+\mathrm{BAO}+\mathrm{CMB}$, Gold $+\mathrm{BAO}+\mathrm{CMB}+$ fgas and Gold $+\mathrm{BAO}+\mathrm{CMB}+$ fgas $+\mathrm{H}(\mathrm{z})$ respectively.

\begin{tabular}{|c|ccc|cc|ccc|c|}
\hline & \multicolumn{3}{|c|}{$\Omega_{m 0}=0.24$} & \multicolumn{3}{c|}{$\Omega_{m 0}=0.28$} & \multicolumn{3}{c|}{$\Omega_{m 0}=0.32$} \\
& $A_{1}$ & $A_{2}$ & $\chi_{\text {Min }}^{2}$ & $A_{1}$ & $A_{2}$ & $\chi_{\text {Min }}^{2}$ & $A_{1}$ & $A_{2}$ & $\chi_{\text {Min }}^{2}$ \\
\hline Gold & $-3.61_{-5.52}^{+4.96}$ & $1.60_{-1.95}^{+2.25}$ & 156.5 & $-3.39_{-5.53}^{+4.97}$ & $1.44_{-1.95}^{+2.25}$ & 156.5 & $-3.17_{-5.54}^{+4.98}$ & $1.27_{-1.95}^{+2.26}$ & 156.5 \\
Gold+BAO & $-4.69_{-5.17}^{+4.55}$ & $2.00_{-1.83}^{+2.14}$ & 158.3 & $-3.01_{-5.09}^{+4.48}$ & $1.30_{-1.13}^{+1.24}$ & 156.7 & $-1.36_{-5.02}^{+4.40}$ & $0.62_{-1.75}^{+2.07}$ & 160.6 \\
$\mathrm{CMB}+\mathrm{BAO}$ & $-1.49_{-2.25}^{+2.52}$ & $0.41_{-0.71}^{+0.72}$ & 0.001 & $-0.21_{-2.58}^{+2.90}$ & $0.14_{-0.79}^{+0.81}$ & 0.001 & $1.08_{-2.91}^{+3.29}$ & $-0.12_{-0.88}^{+0.89}$ & 0.001 \\
$\mathrm{Gold}+\mathrm{CMB}$ & $0.67_{-1.70}^{+1.60}$ & $-0.11_{-0.50}^{+0.57}$ & 161.6 & $-0.33_{-1.96}^{+1.82}$ & $0.20_{-0.59}^{+0.68}$ & 159.1 & $-1.38_{-2.19}^{+2.02}$ & $0.55_{-0.69}^{+0.78}$ & 157.4 \\
$\mathrm{G}+\mathrm{C}+\mathrm{B}$ & $0.11_{-1.60}^{+1.49}$ & $0.04_{-0.48}^{+0.56}$ & 166.1 & $-0.35_{-1.77}^{+1.65}$ & $0.21_{-0.55}^{+0.63}$ & 159.2 & $-0.70_{-1.93}^{+1.79}$ & $0.35_{-0.62}^{+0.71}$ & 160.7 \\
$\mathrm{G}+\mathrm{C}+\mathrm{B}+\mathrm{f}$ & $-0.05_{-1.52}^{+1.42}$ & $0.07_{-0.46}^{+0.54}$ & 192.1 & $-0.53_{-1.69}^{+1.58}$ & $0.25_{-0.54}^{+0.61}$ & 186.3 & $-0.91_{-1.85}^{+1.71}$ & $0.40_{-0.60}^{+0.68}$ & 189.2 \\
$\mathrm{G}+\mathrm{C}+\mathrm{B}+\mathrm{f}+\mathrm{H}$ & $-0.16_{-1.42}^{+1.44}$ & $0.10_{-0.45}^{+0.52}$ & 201.5 & $-0.47_{-1.60}^{+1.54}$ & $0.23_{-0.51}^{+0.57}$ & 195.3 & $-0.73_{-1.71}^{+1.62}$ & $0.33_{-0.56}^{+0.63}$ & 198.8 \\
\hline
\end{tabular}

where $\Omega_{D E}=A_{1}(1+z)+A_{2}(1+z)^{2}+1-\Omega_{m 0}-A_{1}-A_{2}$ [34]. In order to find out whether the constraints from the observations are dependent on the choice of the value of $\Omega_{m 0}$, we give three prior values of $\Omega_{m 0}$ with $\Omega_{m 0}=0.24, \Omega_{m 0}=0.28$ and $\Omega_{m 0}=0.32$. Tables 1 and 2 display constraints on model parameters with 95\% confidence level. From the best fit values given in the Tables one can see that the BAO + CMB alone indicates that the evolving features of dark energy become weaker and weaker with a higher prior matter density, which is the same as obtained in Refs. [33, 34], however the trend is reversed for the Gold $+\mathrm{CMB}$, Gold $+\mathrm{CMB}+\mathrm{BAO}$, Gold $+\mathrm{CMB}+\mathrm{BAO}+$ fgas and Gold $+\mathrm{CMB}+\mathrm{BAO}+$ fgas $+\mathrm{H}(\mathrm{z})$. Meanwhile we find the effects of adding the f-gas and Hubble parameter data sets are not very significant, suggesting that the model parameters are strongly constrained by $\mathrm{CMB}+\mathrm{BAO}+$ Gold. In Fig. 2 we show the constraints on model parameters from all the data sets. The upper and down panels show the constraints on Mod1 and Mod2 respectively. In Fig. 2 the red dot denotes the flat $\Lambda C D M$ model. This figure clearly shows that $\Lambda C D M$ model is consistent with the observations at the $95 \%$ confidence level. In addition one can find that the best fit of model parameters is closest to the $\Lambda C D M$ when $\Omega_{m 0}=0.24$, which can also be seen from the Tables.

In Fig. 3 we plot the evolutionary curves of $w(z)$ for these dark energy models with 
different prior values over $\Omega_{m 0}$. The upper and down panels show the results of Mod1 and Mod2 respectively. In Fig. 3 the solid lines show the evolution of $w(z)$ with the best fit values for the model parameters, and the dotted lines are for $1 \sigma$ and $2 \sigma$ error bars. For Mod1 the best fit curves show that the combination of the data sets considered in this paper favors an evolving dark energy and a crossing of the phantom divide line in the near past, and suggests that the present value of $w$ is very likely less than -1 . Remarkably, these conclusions are almost insensitive to the chosen value of matter density, in a clear contrast to those obtained in [33] where it was found that, when the Sne data are not combined with other observational ones, the evolving property becomes weaker and weaker with higher matter density and the phantom divide may not be crossed with the increasing of matter density. With this being said, it should be pointed out, however, that higher values of $\Omega_{m 0}$ lead to larger errors, especially at the $2 \sigma$ level, as can be seen from the Tables. The results for Mod2 are shown in the down panels of Fig. 3. Unlike Mod1, the best fit curves show that the properties reconstructed depend on the chosen value of matter density. For $\Omega_{m 0}=0.24$, a very mildly evolving dark energy is obtained, but with the increase of $\Omega_{m 0}$ prior considered, the evolving feature of dark energy becomes more evident. This trend is just the opposite to that found in Ref. [34] for just $\mathrm{BAO}+\mathrm{CMB}$ data. These discrepancies can also be found in Tables 1 and 2. However at the $2 \sigma$ confidence level for Mod1 and Mod2 the cosmological constant cannot be ruled out. In addition, we also find that the stringent constraint on $w(z)$ happens around redshift $z \sim 0.5$, which is consistent with that obtained in Refs. [31, 45].

Finally, the evolution of the decelerating parameter $q$ with the redshift is studied for these two parameterized dark energy models with $\Omega_{m 0}=0.28$. The results are shown in Fig. 4 with the left and right panel corresponding to the results of Mod1 and Mod2 respectively. In this figure the dashed lines are the results of $\Lambda C D M$ model with $\Omega_{m 0}=0.28$, the solid lines show the evolution of $q(z)$ when $\Omega_{m 0}=0.28$, the model parameters are the best fit values, and the dotted lines are for $1 \sigma$ errors. The figure shows that the present value of the deceleration parameter $q_{0}$ is less than zero, indicating that the cosmic is undergoing an accelerating expansion, but for different models the value of $q_{0}$ is different. 


\section{CONCLUSION}

In this paper, we have reconstructed the properties of dark energy from recent observational data, including the Gold Sne Ia, the baryonic acoustic oscillation peak from SDSS, the CMB shift parameter, the X-ray gas mass fraction in clusters and the Hubble parameter data. The $\Lambda C D M$ model with curvature and two parameterized dark energy models are discussed. We find that a spatially flat universe is allowed by these data sets at the $68 \%$ confidence level, and a closed universe is slightly favored by the observations. For two parameterized dark energy models, we give the priors on $\Omega_{m 0}$ with $\Omega_{m 0}=0.24, \Omega_{m 0}=0.28$ and $\Omega_{m 0}=0.32$. For the spatially flat case, the constraints on model parameters and the evolutions of $w(z)$ and $q(z)$ are studied. The Gold $+\mathrm{CMB}+\mathrm{BAO}$ give the strong constraints on model parameters. If Mod1 parametrization is used, the best fit curves in Fig. 3 show that the combination of the data sets considered in this paper favors an evolving dark energy, a crossing of the phantom divide line in the near past, and the present value of $w$ being very likely less than -1 . Remarkably, these conclusions are almost insensitive to the chosen value of matter density, in a sharp contrast to those obtained in [33] where the Sne data are not combined with other observational ones. However, the best fit curves in Fig. 3 indicate that the properties of dark energy reconstructed using Mod2 parametrization depend on the chosen value of matter density. For $\Omega_{m 0}=0.24$, a very mildly evolving dark energy is obtained, but with the increase of $\Omega_{m 0}$ prior considered, the evolving feature of dark energy becomes more evident. This trend is just the opposite to that found in Ref. [34] for just $\mathrm{BAO}+\mathrm{CMB}$ data. Therefore, our result seems to suggest that the trend of $\Omega_{m 0}$ dependence for an evolving dark energy is model-dependent. It should be noted, however, that at the $2 \sigma$ confidence level, the cosmological constant are allowed for both Mod1 and Mod2.

\section{Acknowledgments}

This work was supported in part by the National Natural Science Foundation of China under Grants No. 10575035, 10775050 and 10705055, the Program for NCET under Grant No. 04-0784, the Key Project of Chinese Ministry of Education (No. 205110), the Youth Sci-

entific Research Fund of Hunan Provincial Education Department under Grant No. 07B085 
and the Foundation of CSUFT under Grant No. 06Y020.

[1] S. Perlmutter, et al., 1999 Astrophys. J. 517565

[2] A. G. Riess, et al., 1998 Astron. J. 1161009

[3] A. G. Riess, et al., 2004 Astrophys. J. 607665

[4] A. G. Riess et al., 2006 arXiv: astro-ph/0611572

[5] P. Astier et al., 2006 Astron. Astrophys. 44731

[6] W. M. Wood-Vasey et al., 2007 arXiv: astro-ph/0701041

[7] A. Balbi, et al., 2000 Astrophys. J. 545 L1

[8] P. de Bernarbis, et al., 2000 Nature 404955

[9] A. H. Jaffe, et al., 2001 Phys. Rev. Lett. 863475

[10] D. N. Spergel, et al., 2003 Astrophys. J. Supp 148175

[11] D. N. Spergel, et al., 2006 arXiv: astro-ph/0603449

[12] J. A. Peacock, et al., 2001 Nature 410169

[13] D. J. Eisenstein, et al., 2005 Astrophys. J. 633560

[14] T. Padmanabhan, 2006 AIP Conf. Proc. 861179

[15] E. J. Copeland, M. Sami and S. Tsujikawa, 2006 Int. J. Mod. Phys. D 151753

[16] V. Sahni and A. Starobinsky, 2006 Int. J. Mod. Phys. D 152105

[17] L. Perivolaropoulos, 2006 arXiv: astro-ph/0601014

[18] S. Weinberg, 1989 Rev. Mod. Phys. 611

[19] V. Sahni and A. Starobinsky, 2000 Int. J. Mod. Phy. D. 9373

[20] P. J. E. Peebles, B. Ratra, 2003 Rev. Mod. Phys. 75595

[21] T. Padmanabhan, 2003 Phys. Rept. 380235

[22] C. Wetterich, 1988 Nucl. Phys. B 302668

[23] B. Ratra, P. E. J. Peebles, 1988 Phys. Rev. D 373406

[24] R. R. Caldwell, R. Dave, P. J. Steinhardt, 1998 Phys. Rev. Lett. 801582

[25] R. R. Caldwell, 2002 Phys. Lett. B 54523

R. R. Caldwell, M. Kamionkowski, and N. N. Weinberg, 2003 Phys. Rev. Lett. 91071301

S. Nojiri and S. D. Odintsov, 2003 Phys. Lett. B 5711

S. Nojiri and S. D. Odintsov, 2003 Phys. Lett. B 562147 
S. Nojiri and S. D. Odintsov, 2004 Phys. Rev. D 70103522

S. Nojiri, S. D. Odintsov, and S. Tsujikawa, 2005 Phys. Rev. D 71063004

E. Elizalde, S. Nojiri, and S. D. Odintsov, 2004 Phys. Rev. D 70043539

F. Briscese, E. Elizalde, S. Nojiri, S. D. Odintsov, 2007 Phys. Lett. B 646105

S. Nesseris and L. Perivolaropoulos, 2004 Phys. Rev. D 70123529

R. J. Scherrer, 2005 Phys. Rev. D 71063519

F. S. N. Lobo, 2005 Phys. Rev. D 71084011

V. Faraoni, 2005 Class. Quant. Grav. 223235

M. Sami, A. Toporensky, P. V. Tretjakov and S. Tsujikawa, 2005 Phys. Lett. B 619193

L. P. Chimento and R. Lazkoz, 2003 Phys. Rev. Lett. 91211301

R. Cai, A. Wang, 2005 J. Cosmol. Astropart. Phys. 03002

S. M. Carroll, M. Hoffman, and M. Trodden, 2003 Phys. Rev. D 68023509

L. P. Chimento, 2006 Phys. Lett. B 6339

S. K. Srivastava, 2006 hep-th/0605010

E. O. Kahya, V. K. Onemli, 2006 arXiv: gr-qc/0612026

V. K. Onemli, R. P. Woodard, 2004 Phys.Rev. D 70107301

H. Mohseni Sadjadi, 2007 Phys. Lett. B 645108

H. Mohseni Sadjadi, 2006 Phys. Rev. D 73063525

H. Mohseni Sadjadi, M. Alimohammadi, 2006 Phys. Rev. D 74043506

J. C. Fabris, S. V. B. Goncalves, 2006 Phys. Rev. D 74027301

B. McInnes, 2005 Nucl. Phys. B 71855

T. Gonzalez, I. Quiros, 2006 Class. Quant. Grav. 231585

P. Wu and H. Yu, 2006 J. Cosmol. Astropart. Phys. 05008

P. Wu and H. Yu, 2005 Nucl. Phys. B 727355

[26] B. Feng, X. Wang and X. Zhang, 2005 Phys. Lett. B 60735

B. Feng, M. Li, Y. Piao and X. Zhang, 2004 arXiv: astro-ph/0407432

Z. K. Guo, Y. S. Piao, X. M. Zhang and Y. Z. Zhang, 2005 Phys. Lett. B 608177

P. Wu and H. Yu, 2005 Int. J. Mod. Phy. D. 141873

Z. Guo, Y. Piao, X. Zhang and Y. Zhang, 2006 Phys. Rev. D 74127304

R. Lazkoz, G. Leon, I. Quiros, 2007 arXiv: astro-ph/0701353

R. Lazkoz, G. Leon, 2006 Phys. Lett. B 638303 
H. Wei and S. Zhang, 2007 arXiv:0705.4002v1 [gr-qc]

[27] P. Astier, 2000 arXiv:astro-ph/0008306

J. Weller and A. Albrecht, 2002 Phys. Rev. D 65103512

I. Maor, R. Brustein, J. McMahon and P. J. Steinhardt, 2002 Phys. Rev. D 65123003

[28] M. Chevallier, D. Polarski, 2001 Int. J. Mod. Phy. D. 10213

E. V. Linder, 2003 Phys. Rev. Lett. 90091301

[29] H. K. Jassal, J .S. Bagla and T. Padmanabhan, 2005 Mon. Not. Roy. Astron. Soc. 356 L11

[30] V. Sahni, T. D. Saini, A. A. Starobinsky and U. Alam, 2003 JETP lett. 77201

U. Alam, V. Sahni, T. D. Saini and A. A. Starobinsky, 2003 Mon. not. Roy. Ast. Soc. 344 1057

U. Alam, V. Sahni, T. D. Saini and A. A. Starobinsky, 2004 astro-ph/0406672

[31] U. Alam, V. Sahni, T. D. Saini and A. A. Starobinsky, 2004 Mon. Not. Roy. Ast. Soc. 354 275

[32] C. Wetterich, 2004 Phys. Lett. B 59417

P. Wu and H. Yu, 2006 Phys. Lett. B 643315

[33] S. Nesseris and L. Perivolaropoulos, 2007 J. Cosmol. Astropart. Phys. 01018

[34] U. Alam, V. Sahni and A. Starobinsky, 2007 J. Cosmol. Astropart. Phys. 02011

[35] J. R. Bond, G. Efstathiou and M. Tegmark, 1997 Mon. Not. Roy. Astron. Soc. 291 L33

[36] W. Hu and N. Sugiyama 1996 Astrophys. J. 471542

[37] Y. Wang and P. Mukherjee, 2006 Astrophys. J. 6501

[38] S. W. Allen, R. W. Schmidt and A. C. Fabian, 2002 Mon. Not. Roy. Ast. Soc. 334 L11

[39] S. W. Allen, R. W. Schmidt, H. Ebeling, A. C. Fabian and L. van Speybroeck, 2004 Mon. Not. Roy. Ast. Soc. 353457

[40] R. G. Abraham, et al., 2004 Astron. J. 1272455

[41] J. Dunlop, et al., 1996 Nature 381581

H. Spinrad, et al., 1997 Astrophys. J. 484581

T. Treu, et al., 2001 Mon. Not. Roy. Ast. Soc. 326221

T. Treu, et al., 2002 Astrophys. J. Lett 564 L13

P. L. Nolan, et al., 2003 Astrophys. J. 597615

[42] J. Simon, L. Verde and R. Jimenez, 2005 Phys. Rev. D 71123001

[43] R. Jimenez, L. Verde, T. Treu and D. Stern, 2003 Astrophys. J. 593622 
Z. Yi and T. Zhang, 2007 Mod. Phys. Lett. A 2241

L. Samushia and B. Ratra, 2006 Astrophys. J. 650 L5

H. Zhang and Z. Zhu, 2007 astro-ph/0703245

P. Wu and H. Yu, 2007 Phys. Lett. B 64416

H. Wei and S. N. Zhang, 2007 Phys. Lett. B 6447

H. Wei and S. N. Zhang, 2007 arXiv: 0704.3330 [astro-ph]

A. Kurek and M. Szydlowski, 2007 astro-ph/0702484

R. Lazkoz and E. Majerotto, 2007 J. Cosmol. Astropart.Phys. 07015

P. Wu and H. Yu, 2007 J. Cosmol. Astropart. Phys. 03015

A. A. Sen and R. J. Scherrer, 2007 astro-ph/0703416

L. I. Xu, C. W. Zhang, B. R. Chang and H. Y. Liu, 2007 astro-ph/0701519

S. Yin, B. Wang, E. Abdalla and C. Lin, 2007 arXiv:0708.0992[hep-th]

H. Wan, Z. Yi and T. Zhang, 2007 arXiv:0706.2737[astro-ph]

J. He, B. Wang and E. Papantonopoulos, 2007 arXiv:0707.1180[gr-qc]

C. Feng, B. Wang, Y. Gong and R. Su, 2007 arXiv:0706.4033[astro-ph]

[44] S. Nesseris and L. Perivolaropoulos, 2004 Phys. Rev. D 70043531

[45] Y. G. Gong, 2005 Class. Quantum Grav. 222121

Y. G. Gong and Y.Z. Zhang, 2005 Phys. Rev. D 72043518

M. Chevallier and D. Polarski, 2001 Int. J. Mod. Phys. D 10213

Y. Gong and A. Wang, 2007 Phys. Rev. D 75043520 


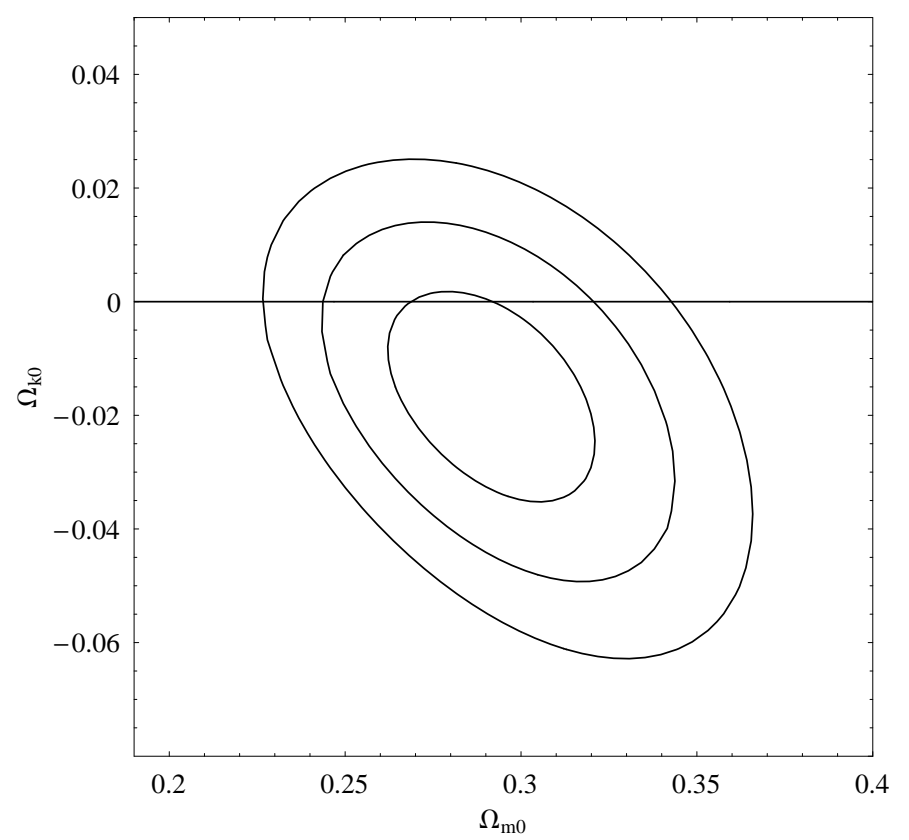

FIG. 1: The $1 \sigma, 2 \sigma$ and $3 \sigma$ confidence contours for a $\Lambda C D M$ universe with curvature from the combination of Sne Ia, BAO, CMB, the X-ray gas mass fraction in clusters and Hubble parameter data. 

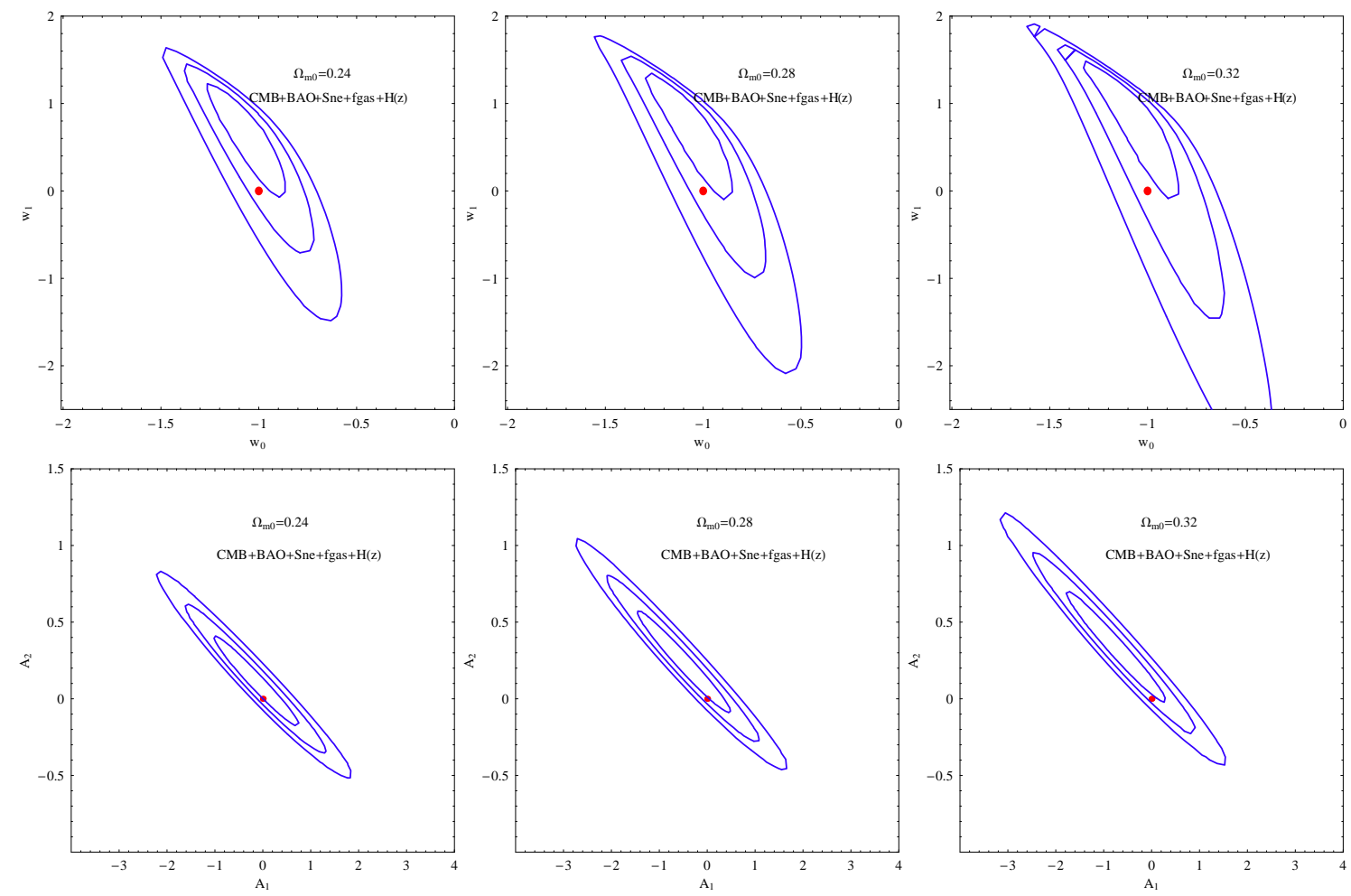

FIG. 2: The constraints on two parameterized dark energy models from the combination of the Gold Sne Ia, BAO, CMB, the X-ray gas mass fraction in clusters and the Hubble parameter data. The upper and down panels, respectively, show the results of $w(z)=w_{0}+w_{1} z /(1+z)(\operatorname{Mod} 1)$ and $w(z)=\frac{1+z}{3} \frac{A_{1}+2 A_{2}(1+z)}{\Omega_{D E}}-1(\operatorname{Mod} 2)$ with three different priors over $\Omega_{m 0}: \Omega_{m 0}=0.24, \Omega_{m 0}=0.28$ and $\Omega_{m 0}=0.32$. The red dot represents the $\Lambda C D M$ model. 

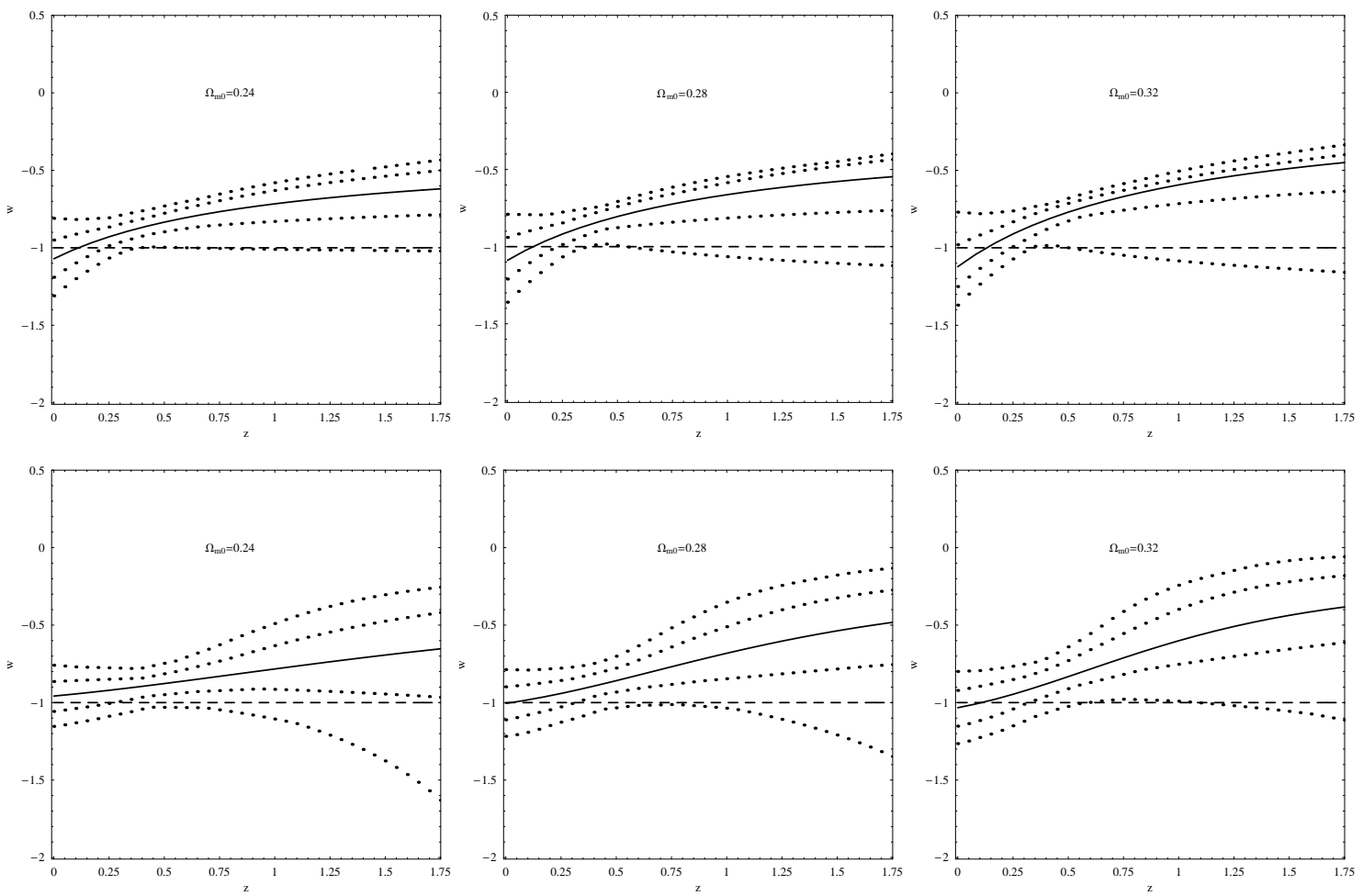

FIG. 3: The evolution of $w(z)$. The upper and down panels, respectively, show the results of Mod1 and Mod2 with $\Omega_{m 0}=0.24, \Omega_{m 0}=0.28$ and $\Omega_{m 0}=0.32$. The solid line shows the evolution of $w(z)$ with the model parameters at the best fit values, and the dotted lines are for the $1 \sigma$ and $2 \sigma$ errors.
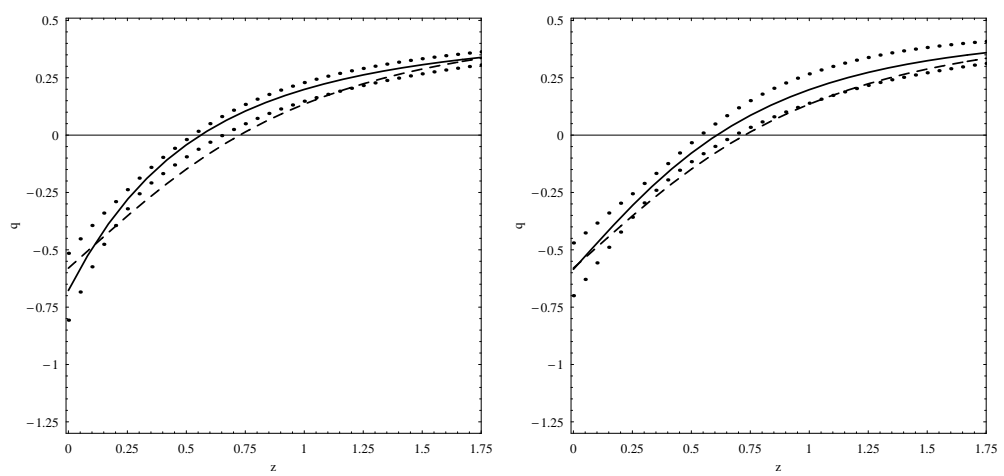

FIG. 4: The behavior of $q(z)$. The left and right panel, respectively, shows the results of Mod1 and Mod2 with $\Omega_{m 0}=0.28$. The dashed line represents the $\Lambda C D M$ with $\Omega_{m 0}=0.28$, the solid line shows the evolution of $q(z)$ with $\Omega_{m 0}=0.28$ and the model parameters at the best fit values, and the dotted lines are for the $1 \sigma$ error. 